\title{
Increased serum levels of LL37, HMGB1 and S100A9 during exacerbation in COPD patients
}

\author{
To the Editor:
}

Chronic obstructive pulmonary disease (COPD) is a severe and progressive lung disease characterised by destruction of lung parenchyma and chronic airway inflammation [1]. A major cause of COPD is chronic exposure to noxious gases and particles, including cigarette smoke. During exacerbation, COPD patients experience a worsening of symptoms that coincides with increased inflammation and accelerated decline in lung function, resulting in a decrease in quality of life and increased healthcare costs. Approximately half of the COPD exacerbations causing hospitalisation are associated with respiratory viral and/or bacterial infections [2]. However, the underlying mechanisms causing COPD exacerbations are unknown [3]. Molecules derived from viruses and bacteria during airway infection can trigger the activation of pattern recognition receptors (PRRs) on lung structural and innate immune cells, and may thus contribute to the aggravation of inflammation during COPD exacerbations [1]. Interestingly, damage-associated molecular patterns (DAMPs) released from damaged or necrotic cells are also known to activate PRRs, including Toll-like receptors (TLRs) and the receptor for advanced glycation end-products (RAGE) [4]. A role for DAMPs has been proposed in the pathogenesis of COPD, as various DAMPs have been found to be increased in lung fluids and serum of COPD patients $[5,6]$. Furthermore, Ager, the gene encoding RAGE, has been identified by genome-wide association studies as a susceptibility gene for COPD [7, 8]. Moreover, the serum levels of soluble RAGE (sRAGE), a decoy receptor for RAGE, were shown to be significantly lower in COPD patients, while the RAGE ligand EN-RAGE (also known as S100 calcium-binding protein (S100)A12) was significantly higher in COPD patients compared with smoking and nonsmoking controls [9]. It is currently unknown, however, whether DAMPs play a role in COPD exacerbations. Here, we hypothesised that the release of DAMPs is increased during exacerbations of COPD.

To address our hypothesis, we performed a post hoc analysis on samples collected in a prospective randomised controlled trial on COPD exacerbations. We used a cohort of COPD patients with relatively mild disease who discontinued the use of corticosteroids or long-acting $\beta_{2}$-agonists and had stable disease for $\geq 2$ months after discontinuation [10]. Patient characteristics are summarised in figure $1 \mathrm{k}$ and the study design has been extensively described by BATHOORN et al. [3]. Serum and induced sputum samples were collected when the patients reported an exacerbation to the outpatient clinic and in the same patients during stable disease. Viral infection status was determined by detection of viral respiratory pathogens in sputum by panel-based real-time PCR used for routine diagnostic purposes. Bacterial infection status was assessed using an algorithm to interpret conventional sputum culture results as described previously [3]. 11 of the total of 40 patients with an exacerbation were found to be positive for airway infection, of which three patients had a viral infection, six patients had a bacterial infection and two patients had both a viral and a bacterial infection. A panel of six DAMPs, grossly grouped into TLR2-activating DAMPs (galectin-3 and high-mobility group box (HMGB)1), TLR4-activating DAMPs (HMGB1 and S100A9), TLR9-activating DAMPs (double-stranded (ds)DNA and mitochondrial (mt)DNA) and RAGE-activating DAMPs (LL37, HMGB1 and S100A9) [4,5], and sRAGE were measured in serum $(\mathrm{n}=40)$ and induced sputum $(n=35)$ of COPD patients in stable disease and during exacerbation, using commercially available ELISA kits (galectin-3, S100A9 and sRAGE: R\&D Systems, Minneapolis, MN, USA; HMGB1: Chondrex Inc., Redmond, WA, USA; LL37: Hycult Biotech Inc., Plymouth Meeting, PA, USA; dsDNA: PicoGreen, Life Technologies, Carlsbad, CA, USA; detection limits were 62.5, 31.2, 62.5, 800, 140 and $3900 \mathrm{pg} \cdot \mathrm{mL}^{-1}$, respectively) or quantitative PCR as described previously (mtDNA) [11]. All ELISA kits were tested and found suitable for detection of DAMPs in both serum and dithiothreitol-treated sputum samples. All data are presented as mean \pm SEM.

All selected DAMPs were detectable in induced sputum and serum, both in stable phase and during exacerbation. The serum levels of the RAGE-activating DAMPs LL37, HMGB1 and S100A9 were 

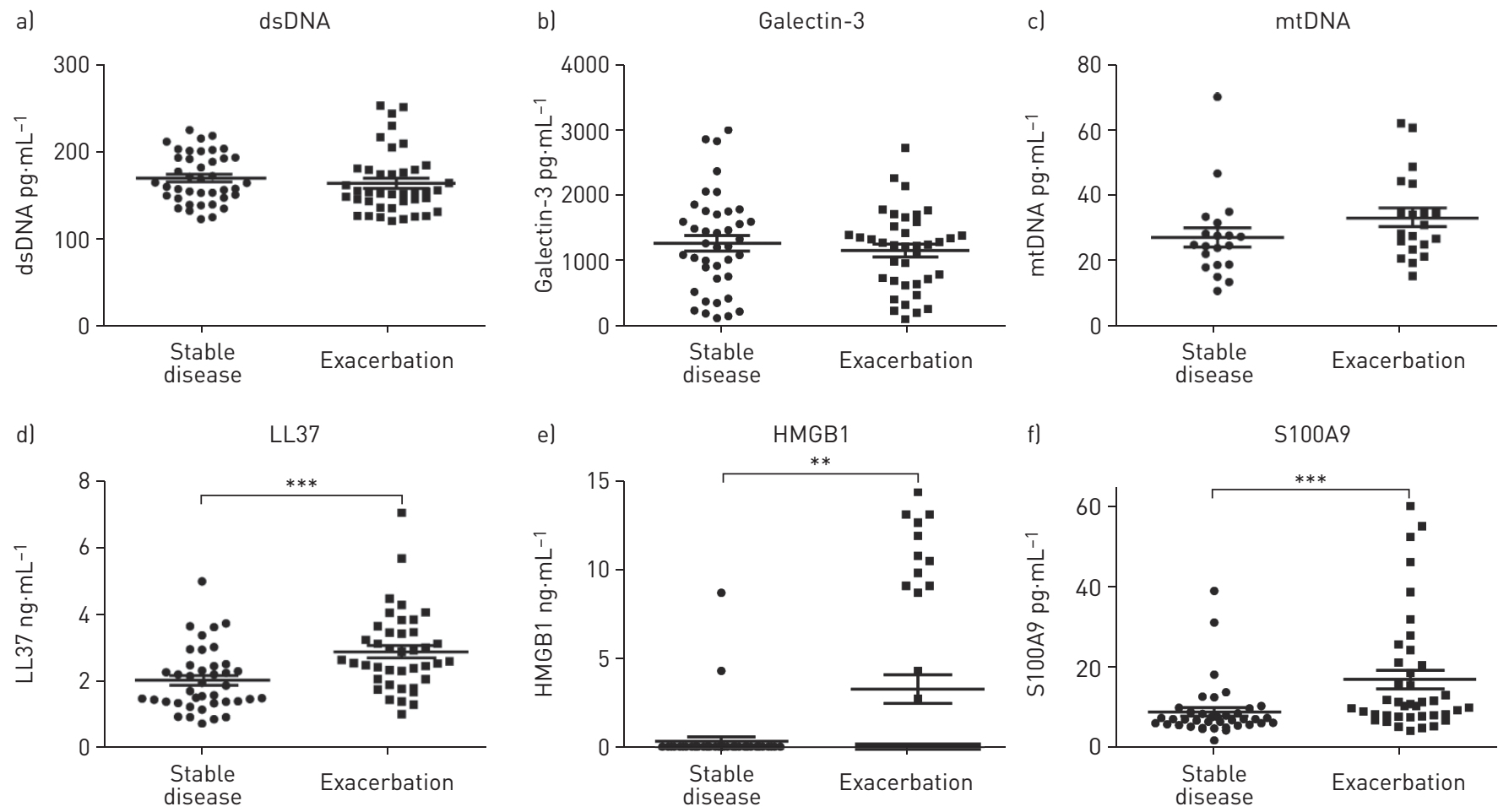

g)

h) HMGB1

i)

S100A9
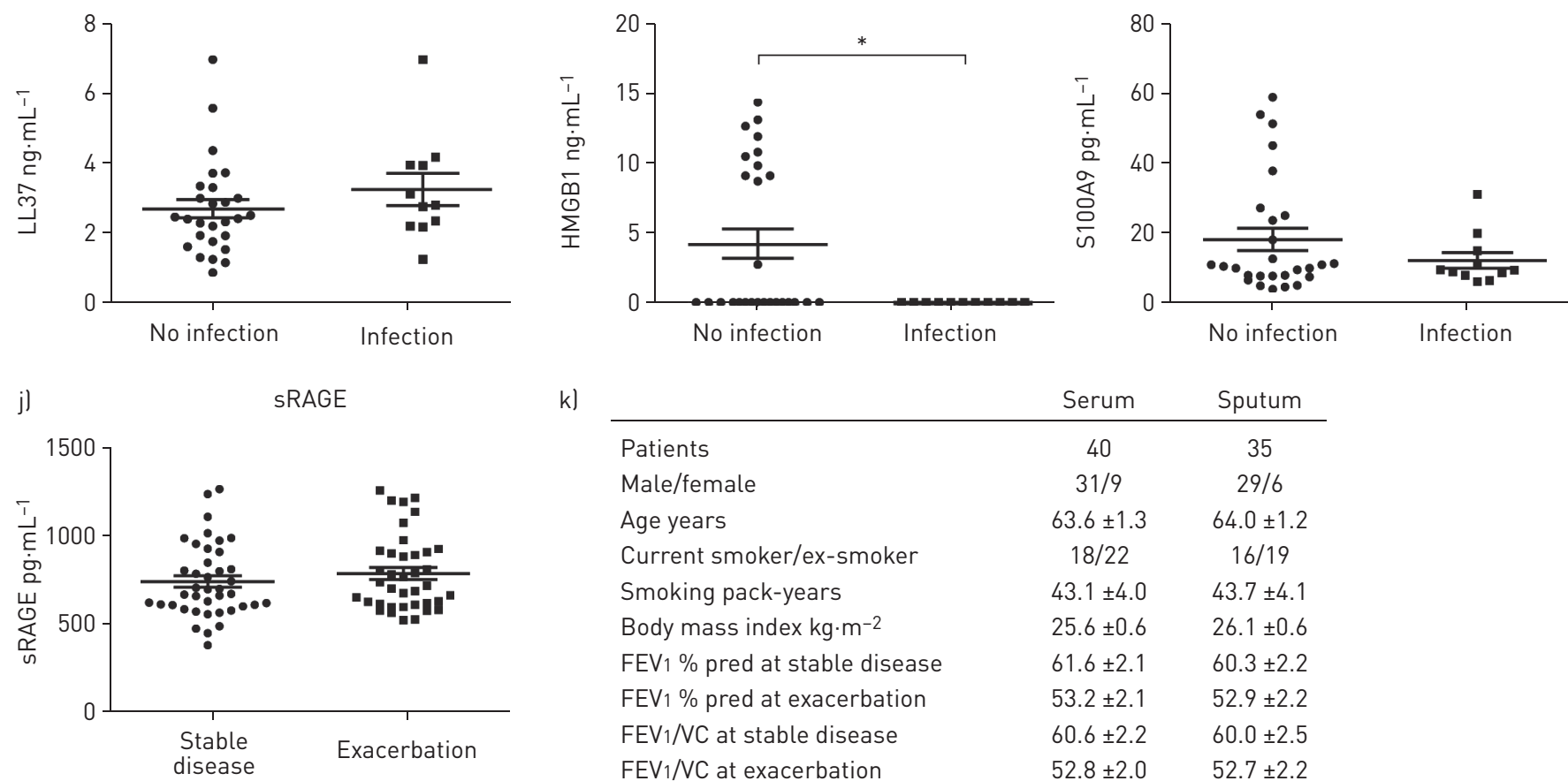

k)

\begin{tabular}{lcc} 
& Serum & Sputum \\
\hline Patients & 40 & 35 \\
Male/female & $31 / 9$ & $29 / 6$ \\
Age years & $63.6 \pm 1.3$ & $64.0 \pm 1.2$ \\
Current smoker/ex-smoker & $18 / 22$ & $16 / 19$ \\
Smoking pack-years & $43.1 \pm 4.0$ & $43.7 \pm 4.1$ \\
Body mass index $\mathrm{kg} \cdot \mathrm{m}^{-2}$ & $25.6 \pm 0.6$ & $26.1 \pm 0.6$ \\
FEV $1 \%$ pred at stable disease & $61.6 \pm 2.1$ & $60.3 \pm 2.2$ \\
FEV $1 \%$ pred at exacerbation & $53.2 \pm 2.1$ & $52.9 \pm 2.2$ \\
FEV $1 /$ VC at stable disease & $60.6 \pm 2.2$ & $60.0 \pm 2.5$ \\
FEV $1 /$ VC at exacerbation & $52.8 \pm 2.0$ & $52.7 \pm 2.2$ \\
Bacterial/viral infection & $8 / 5$ & $8 / 5$
\end{tabular}

FIGURE 1 The levels of S100 calcium-binding protein (S100)A9, high-mobility group box (HMGB)1 and LL37 are increased in serum of chronic obstructive pulmonary disease (COPD) patients during exacerbation. Levels of the damage-associated molecular patterns a) double-stranded (ds)DNA, b) galectin-3, c) mitochondrial (mt)DNA, d) LL37, e) HMGB1 and f) S100A9 in serum of COPD patients $(\mathrm{n}=40)$ in stable disease and during exacerbation. Levels of g) LL37, h) HMGB1 and i) S100A9 during exacerbation in serum of patients with and without airway infection (either bacterial or viral). j) Serum levels of the decoy receptor sRAGE (soluble receptor for advanced glycation end-products) in COPD patients in stable disease and during exacerbation. Horizontal lines and whiskers represent the mean and SEM, respectively. Significance between stable disease and exacerbation was tested using the Wilcoxon signed-rank test Significance between COPD patients with and without airway infection was tested using the Mann-Whitney U-test. ${ }^{*}: \mathrm{p} \leq 0.05 ;{ }^{* *}: \mathrm{p} \leq 0.01 ;{ }^{* * *}: \mathrm{p} \leq 0.001$. k) Patient characteristics. Data are presented as $n$ or mean \pm SEM. FEV 1 : forced expiratory volume in $1 \mathrm{~s}$; VC: vital capacity. 
significantly increased during exacerbation compared with stable disease, while no significant differences were found in the levels of dsDNA, mtDNA and galectin-3 (fig. 1a-f). The levels of DAMPs in serum did not correlate with either blood neutrophil counts or total leukocyte counts, indicating that the increased DAMP levels are not caused by increased numbers of blood leukocytes during exacerbation (data not shown). Furthermore, in an experiment comparing the DAMP levels in plasma with serum collected from healthy volunteers, no significant differences were detected for dsDNA, LL37 and galectin-3, although the levels of HMGB1 were higher in serum compared with plasma (data not shown). These results indicate that the increased DAMP levels in serum of COPD patients during exacerbation are not an artefact from the coagulation process during serum preparation. In sputum, no significant differences were detected between stable disease and exacerbation for dsDNA $\left(558.3 \pm 39.3\right.$ and $\left.524.8 \pm 37.2 \mathrm{pg} \cdot \mathrm{mL}^{-1}\right)$, galectin-3 $\left(1.14 \pm 0.0\right.$ and $\left.1.19 \pm 0.0 \mathrm{pg} \cdot \mathrm{mL}^{-1}\right)$, HMGB1 $\left(64.3 \pm 6.4\right.$ and $\left.69.7 \pm 5.0 \mathrm{ng} \cdot \mathrm{mL}^{-1}\right)$, LL37 $(2.0 \pm 0.3$ and $\left.2.0 \pm 0.4 \mathrm{ng} \cdot \mathrm{mL}^{-1}\right)$, mtDNA $\left(14.0 \pm 9.6\right.$ and $\left.24.0 \pm 15.0 \mathrm{ng} \cdot \mathrm{mL}^{-1}\right)$ and S100A9 $\left(0.79 \pm 0.1\right.$ and $0.65 \pm 0.1 \mathrm{pg} \cdot \mathrm{mL}^{-1}$; all during stable disease and exacerbation, respectively).

In serum samples, two subgroups of patients were observed for HMGB1, with one group showing increased levels of HMGB1 during exacerbation and the other group maintaining undetectable levels of HMGB1, i.e. $<0.8 \mathrm{ng} \cdot \mathrm{mL}^{-1}$. Notably, females had significantly higher levels of HMGB1 $\left(2.3 \pm 0.8 \mathrm{ng} \cdot \mathrm{mL}^{-1}\right.$ for males and $7.2 \pm 2.0 \mathrm{ng} \cdot \mathrm{mL}^{-1}$ for females; $\left.\mathrm{p}=0.01\right)$ and $\mathrm{S} 100 \mathrm{~A} 9\left(13.5 \pm 2.0 \mathrm{pg} \cdot \mathrm{mL}^{-1}\right.$ for males and $28.6 \pm 6.7 \mathrm{pg} \cdot \mathrm{mL}^{-1}$ for females; $\mathrm{p}=0.01$ ) during exacerbation compared with males. Furthermore, we observed that none of the COPD patients with a proven bacterial or viral infection showed increased levels of HMGB1 (fig. $1 \mathrm{~h}$ ) or S100A9 (fig. 1i) during exacerbation, with a significant difference in HMGB1 levels between COPD patients with and without airway infection during exacerbation. Thus, our data suggest that infection status does not contribute to increased release of HMGB1 and S100A9 during exacerbations. For LL37, no association with airway infection status was found (fig. 1g). Since we expected that PRR activation might play a role in the aggravation of airway inflammation during exacerbation, it is tempting to speculate that DAMPs contribute to PRR-induced inflammatory responses during exacerbation, especially in those patients who do not have airway infections.

Interestingly, all DAMPs that we found to be increased in serum of COPD patients during exacerbation are ligands of the RAGE receptor, suggesting that exacerbation may lead to increased RAGE signalling. However, most studies that suggest RAGE as a receptor for LL37 provide indirect evidence [5, 12, 13]. Nevertheless, we observed that the LL37-induced interleukin- 8 production by human bronchial epithelial cells can be partly inhibited by RAGE-antagonistic peptide (data not shown). Therefore, it will be of interest to further elucidate the role of RAGE in exacerbations of COPD. Multiple studies have shown that sRAGE is decreased in serum or plasma of COPD patients, which may act to further potentiate the effect of RAGE ligands [9, 14-16]. However, in our study, induced sputum and serum levels of sRAGE did not differ between stable disease and exacerbation (sputum levels $159.5 \pm 18.7$ and $164.0 \pm 21.5 \mathrm{pg} \cdot \mathrm{mL}^{-1}$, respectively) (fig. 1j). Similarly, we did not observe a correlation between decreased lung function and serum or sputum levels of sRAGE (fig. 1k), as previously reported [14-16].

In conclusion, we show that, within a panel of six DAMPs, the RAGE-activating DAMPs LL37, HMGB1 and S100A9 are increased in serum of COPD patients during exacerbation. The increase in S100A9 and HMGB1 was associated positively with the female sex and negatively with infection status. Although none of the DAMPs were increased in sputum, serum levels of HMGB1, S100A9 and LL37 may be useful in the clinical detection of COPD exacerbations unrelated to infection. However, it will be of importance to confirm our findings in a clinical cohort of COPD patients with more severe disease. Furthermore, our data suggest that these specific DAMPs may play a role in the pathogenesis of COPD exacerbations, potentially by activation of RAGE. Therefore, it will be of further interest to explore whether this receptor may constitute a target for novel therapeutic strategies in COPD exacerbations.

@ERSpublications

Serum levels of the RAGE-activating DAMPs LL37, HMGB1 and S100A9 are increased during exacerbation in COPD patients http://ow.ly/Idh3r

\footnotetext{
Simon D. Pouwels ${ }^{1,2}$, Martijn C. Nawijn ${ }^{1,2}$, Erik Bathoorn ${ }^{3}$, Annelies Riezebos-Brilman ${ }^{3}$, Antoon J.M. van Oosterhout ${ }^{1,2}$, Huib A.M. Kerstjens ${ }^{2,4}$ and Irene H. Heijink ${ }^{1,2,4}$

${ }^{1}$ Dept of Pathology and Medical Biology, Experimental Pulmonology and Inflammation Research, University of Groningen, University Medical Center Groningen, Groningen, The Netherlands. ${ }^{2}$ GRIAC Research Institute, University of Groningen, University Medical Center Groningen, Groningen, The Netherlands. ${ }^{3}$ Dept of Medical Microbiology, University of Groningen, University Medical Center Groningen, Groningen, The Netherlands. ${ }^{4}$ Dept of Pulmonology, University of Groningen, University Medical Center Groningen, Groningen, The Netherlands.
}

Correspondence: Simon D. Pouwels, Dept of Pathology and Medical Biology, Experimental Pulmonology and Inflammation Research, UMCG, Hanzeplein 1, 9713 GZ Groningen, The Netherlands. E-mail: s.d.pouwels@umcg.nl 
Received: Aug 292014 | Accepted after revision: Jan 192015 | First published online: March 052015

Support statement: This study was funded by The Netherlands Asthma Foundation (project 3.2.11.025) and Stichting Astma Bestrijding (project 2013/008). Funding information for this article has been deposited with FundRef.

Conflict of interest: None declared.

\section{References}

1 Brusselle GG, Joos GF, Bracke KR. New insights into the immunology of chronic obstructive pulmonary disease. Lancet 2011; 378: 1015-1026.

2 Decramer M, Janssens W, Miravitlles M. Chronic obstructive pulmonary disease. Lancet 2012; 379: 1341-1351.

3 Bathoorn E, Liesker JJ, Postma DS, et al. Change in inflammation in out-patient COPD patients from stable phase to a subsequent exacerbation. Int J Chron Obstruct Pulmon Dis 2009; 4: 101-109.

4 Chen GY, Nuñez G. Sterile inflammation: sensing and reacting to damage. Nat Rev Immunol 2010; 10: 826-837.

5 Pouwels SD, Heijink IH, ten Hacken $\mathrm{NH}$, et al. DAMPs activating innate and adaptive immune responses in COPD. Mucosal Immunol 2014; 7: 215-226.

6 Pouwels SD, Heijink $\mathrm{IH}$, van Oosterhout AJ, et al. A specific DAMP profile identifies susceptibility to smoke-induced airway inflammation. Eur Respir J 2014; 43: 1183-1186.

7 Repapi E, Sayers I, Wain LV, et al. Genome-wide association study identifies five loci associated with lung function. Nat Genet 2010; 42: 36-44.

8 Castaldi PJ, Cho MH, Litonjua AA, et al. The association of genome-wide significant spirometric loci with chronic obstructive pulmonary disease susceptibility. Am J Respir Cell Mol Biol 2011; 45: 1147-1153.

9 Cockayne DA, Cheng DT, Waschki B, et al. Systemic biomarkers of neutrophilic inflammation, tissue injury and repair in COPD patients with differing levels of disease severity. PLoS One 2012; 7: e38629.

10 Bathoorn E, Liesker JJ, Postma DS, et al. Anti-inflammatory effects of combined budesonide/formoterol in COPD exacerbations. COPD 2008; 5: 282-290.

11 Zhang Q, Raoof M, Chen Y, et al. Circulating mitochondrial DAMPs cause inflammatory responses to injury. Nature 2010; 464: 104-107.

12 Roundy LM, Jia W, Zhang J, et al. LL-37 induced cystitis and the receptor for advanced glycation end-products (RAGE) pathway. Adv Biosci Biotechnol 2013; 4: 1-8.

13 Zhang J, Xu X, Rao NV, et al. Novel sulfated polysaccharides disrupt cathelicidins, inhibit RAGE and reduce cutaneous inflammation in a mouse model of rosacea. PLoS One 2011; 6: e16658.

14 Iwamoto $\mathrm{H}$, Gao J, Pulkkinen V, et al. Soluble receptor for advanced glycation end-products and progression of airway disease. BMC Pulm Med 2014; 14: 68.

15 Gopal P, Reynaert NL, Scheijen JL, et al. Association of plasma sRAGE, but not esRAGE with lung function impairment in COPD. Respir Res 2014; 15: 24.

16 Smith DJ, Yerkovich ST, Towers MA, et al. Reduced soluble receptor for advanced glycation end-products in COPD. Eur Respir J 2011; 37: 516-522.

\section{Increased expression of granzymes $A$ and $B$ in fatal asthma}

To the Editor:

Inflammatory mediators other than the classic T-helper cell type 2 (Th2)-related pathway play a role in asthma, especially in severe asthma and in exacerbations [1]. Recent studies have suggested a role for cytotoxic CD8 T-cells in lung function impairment [2], lung function decline [3] and smoking-associated asthma [4].

The granules of effector cytotoxic CD8 cells and natural killer (NK) cells contain a family of serine proteases called granzymes (granule-secreted enzymes) [5]. Granzymes are stored in the cytotoxic granules and require perforin to be delivered into the target cells to exert their cytotoxic function $[5,6]$. Granzymes can also be released to the extracellular space and cleave extracellular matrix components, contributing to remodelling in chronic inflammation $[5,6]$. Granzyme $(\mathrm{Gzm}) \mathrm{A}$, the most abundant granzyme, can activate macrophages to secrete cytokines [6].

$\mathrm{CD}^{+} \mathrm{T}$-cells with increased perforin expression have been identified in the lungs of patients who died of asthma [7]. Several other cell types also express granzymes, and the role of granzymes expressed by these cells other than cytotoxic T-cells and NK cells remains unclear. After allergen challenge, asthmatics 